\title{
Angle Measurement of the Papillary Projection in Unilocular- solid Adnexal Cysts as a Predictor of Malignancy: A Three- dimensional Ultrasound Study
}

\author{
Daniel Sanin-Ramirez ${ }^{1}$, Maria P Sanin ${ }^{2}$, Federico Moreno ${ }^{3}$, Isabel Carriles ${ }^{4}$, Nabil Manzour ${ }^{5}$, Julio Vara ${ }^{6}$, Juan L Alcazar ${ }^{7}$
}

\begin{abstract}
Aim and objective: To analyze the value of the angle between the papillary projection and the cyst's wall for differentiating between benign and malignant adnexal masses using three-dimensional ultrasound.

Materials and methods: Retrospective study between January 2003 and December 2012 including a series of non-consecutive patients diagnosed as having a unilocular-solid mass in ultrasound. Definitive diagnosis was based on a histological result obtained after surgical removal or ultrasonographic follow-up until the disappearance of the mass. In a 3D volume, the angles formed by the solid component and the cyst's wall in both lateral borders were measured in the three planes, obtaining six different angles. The variables analyzed were an average angle, widest angle, narrowest angle, the difference between the widest and narrowest angle, number of obtuse $\left(>90^{\circ}\right)$, and acute $\left(<90^{\circ}\right)$ angles.

Results: Eighty-one patients were included. The patient's mean age was 44.2 years. Twenty-four (29.6\%) of them were postmenopausal and 57 (70.4\%) were premenopausal. The mean average angle, widest angle, narrowest angle, the difference between the widest and narrowest angle, number of obtuse and acute angles in benign masses were $81.5^{\circ}, 105.3^{\circ}, 58^{\circ}, 47.3^{\circ}, 2.3$, and 3.7 , respectively. These figures in malignant masses were $75.7^{\circ}, 103.7^{\circ}, 49.1^{\circ}, 54.6,1^{\circ}, 1.8$, and 4.2 , respectively. We did not find statistical significance in any comparison.

Conclusion: Measurement of the angles between the solid component and the cyst's wall in unilocular-solid adnexal masses with only one papillary projection is not useful as a predictor of malignancy.
\end{abstract}

Keywords: Adnexal mass, Ovarian cancer, Three-dimensional, Ultrasound and diagnosis.

Donald School Journal of Ultrasound in Obstetrics and Gynecology (2020): 10.5005/jp-journals-10009-1664

\section{INTRODUCTION}

The differential diagnosis of adnexal masses is challenging but crucial for the proper management of patients with this condition. Transvaginal ultrasonography is an excellent tool for this purpose. An experienced sonographer, using pattern recognition with grayscale and Doppler ultrasound can achieve a sensitivity of $88-96 \%$ and a specificity of $90-96 \%$ in the distinction of benign and malignant tumors. ${ }^{1-4}$

Some lesions like a simple cyst, endometrioma, dermoid cyst, or hydrosalpinx are easy to characterize with a good degree of certainty. 5,6 These lesions are considered as simple "instant" diagnosis for the International Ovarian Tumor Analysis (IOTA) group. ${ }^{7}$ The use of the so-called ultrasound-based simple rules developed by IOTA also makes some benign lesions easier to diagnose. $^{8}$

However, even an expert sonographer cannot differentiate a benign mass from a malignant one in about $7 \%$ of cases and logistic regression models do not seem to help with this distinction. ${ }^{9}$ Some examples of the latter are masses with papillary projections, multilocular cysts with more than 10 locules, cysts with low-level echogenicity of cyst fluid and masses with moderate color score. ${ }^{5}$ Taking into account the histological diagnosis, borderline tumors, papillary cystadenofibromas, struma ovarii, and some myomas correspond to these lesions difficult to diagnose. ${ }^{5}$

Unilocular cysts with papillary projections constitute only approximately $7 \%$ of all adnexal masses but are a focus of interest because of their diagnostic difficulty. ${ }^{10}$ Some authors have proposed analyzing specific parameters regarding the ultrasonographic characteristics of these lesions, including the number of papillary
${ }^{1}$ Department of Obstetrics and Gynecology, Pontifical Bolivarian University, Medellin, Colombia

${ }^{2}$ Department of Radiology, Antioquia University, Medellin, Colombia

${ }^{3}$ Department of Medicine, CES University, Medellin, Colombia

${ }^{4-7}$ Department of Obstetrics and Gynecology, Clinica Universidad de Navarra, Pamplona, Spain

Corresponding Author: Juan L Alcázar, Department of Obstetrics and Gynecology, Clinica Universidad de Navarra, Pamplona, Spain, Phone: +34-948-296234, e-mail: jlalcazar@unav.es

How to cite this article: Sanin-Ramirez D, Sanin MP, Moreno F, et al. Angle Measurement of the Papillary Projection in Unilocular-solid Adnexal Cysts as a Predictor of Malignancy: A Three-dimensional Ultrasound Study. Donald School J Ultrasound Obstet Gynecol 2020;14(4):311-314.

Source of support: Nil

Conflict of interest: None

projections, the presence of blood flow, the size, and the surface of the solid component. ${ }^{10}$

One additional specific parameter assessed is the angle formed between the cyst's wall and the solid component. To date, only two studies have taken into account this last parameter to characterize adnexal masses, but their results are controversial. ${ }^{11,12}$ In these studies, the authors assessed the angle using a 2D image. However, a solid component is a three-dimensional structure. Three-dimensional ultrasound allows displaying the structure understudy in the three orthogonal planes. ${ }^{13}$ 
We hypothesized that three-dimensional ultrasound could be more accurate for measuring the angle formed between the cyst's wall and the solid component more comprehensively. This study aims to analyze the value of the angle between the papillary projection and the cyst's wall for differentiating between benign and malignant adnexal masses using three-dimensional ultrasound.

\section{Materials and Methods}

This is a retrospective study including a series of non-consecutive patients diagnosed as having a unilocular-solid mass in ultrasound evaluated and treated at a tertiary university hospital. Patients were evaluated between January 2003 and December 2012. Patients were identified searching in the ultrasound database. Clinical, ultrasound, and histological data were retrieved reviewing patients' records. Institutional Review Board was waived due to the nature of the study design. All women provided informed consent for using their data for research purposes.

Inclusion criteria were as follows:

- Patients aged between 18 days and 90 years.

- Diagnosis of unilocular-solid adnexal cysts with only one papillary projection identified with transvaginal ultrasound, whose 3D volume was available for evaluation. In cases of bilateral mass, the one with the largest size was analyzed.

- Definitive diagnosis is based on the histological result obtained after surgical removal in our institution or ultrasonographic follow-up until the disappearance of the mass.

The exclusion criteria were as follows: pregnant patients at the time of diagnosis, presence of ascites or peritoneal carcinomatosis, or patients whose follow-up did not result in the disappearance of the lesion. We also excluded those cases in whom the whole mass was not included within the 3D volume.

All patients had been evaluated with a Voluson E8 or 730 Expert system (GE Healthcare, Zipf, Austria) equipped with a 5-9 MHz endovaginal probe with 3D sonographic capabilities. A $3 \mathrm{D}$ volume was captured including the whole tumor; the image capture lasted from 2 to 6 seconds depending on the size of the 3D box. The volume was stored for posterior processing using virtual organ computer-aided analysis (VOCAL) software (GE Healthcare, Zipf, Austria).

As stated above, we used 3D volumes of the unilocular-solid adnexal cysts with only one papillary projection. A unilocularsolid adnexal cyst was defined as an anechoic cyst with a solid

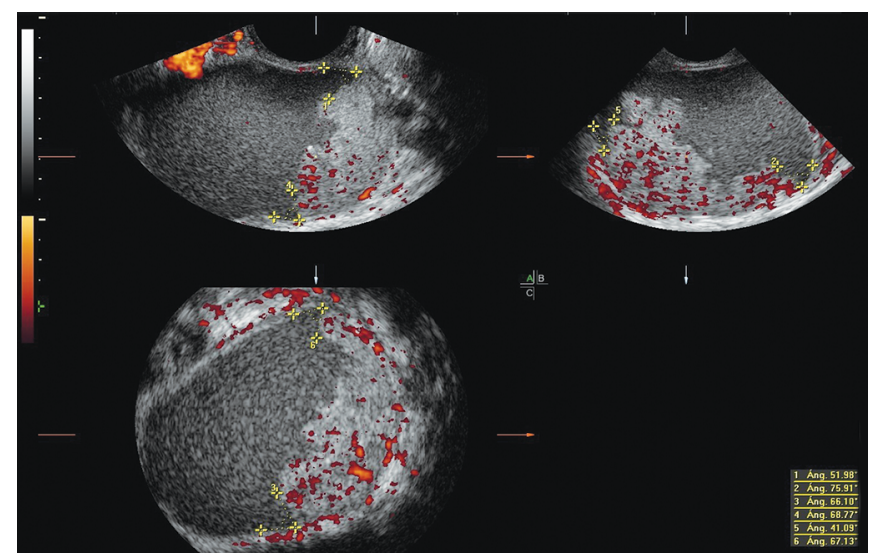

Fig. 1: All acute angles in a borderline ovarian tumor of a premenopausal woman component within the mass $>3 \mathrm{~mm} .{ }^{14}$ A solid papillary projection was defined as an echoic focal structure protruding inside the cystic component of the mass. ${ }^{14}$ Virtual navigation was done throughout the whole tumor in three orthogonal planes to obtain the largest dimension of the solid component in plane A. The angles formed by the solid component and the cyst's wall in both lateral borders were measured in each plane (A, B, and C), obtaining six different angles (Figs 1 and 2). The variables analyzed were average angle (from the six angles obtained), widest angle, narrowest angle, the difference between the widest and narrowest angle, number of obtuse $\left(>90^{\circ}\right)$, and acute $\left(<90^{\circ}\right)$ angles. All measurements were performed by one single examiner.

The Kolmogorov-Smirnov test was used to assess the normal distribution of continuous data. Continuous variables are presented as mean and standard deviation and the range since all data fitted normal distribution. Categorical results are presented as number of cases and percentages. Continuous data were compared with the use of the one-way analysis of variance. To determine the best score cutoff value to discriminate between malignant and benign adnexal masses, a receiver operating characteristic $(\mathrm{ROC})$ curve was plotted.

A significance level of $<0.05$ was used in all tests. All statistical procedures were carried with SPSS software version 21.0 (IBM SPSS Statistics, Chicago, Illinois, USA).

\section{Results}

Eighty-one patients with unilocular-solid adnexal cysts were included in this study. The patient's mean age was 44.2 years (SD: 13.5 -range 18-85). Twenty-four (29.6\%) of them were postmenopausal and 57 (70.4\%) were premenopausal. Sixty-three (77.8\%) lesions were surgically removed, so histological diagnosis was available, whereas 18 (22.2\%) of them were followed up until spontaneous resolution occurred, after 3-6 months. The latter were considered benign for analytical purposes.

Out of all the masses, 52 (64.2\%) were benign and 29 (35.8\%) were malignant; of which 21 (25.9\%) were primary invasive ovarian tumors, 7 (8.6\%) borderline, and 1 (1.2\%) was a metastasis.

The results of the comparison of values of average angle, widest angle, narrowest angle, the difference between the widest and narrowest angle, number of obtuse $\left(>90^{\circ}\right)$ and acute $\left(<90^{\circ}\right)$ angles between benign and malignant masses are illustrated in Table 1.

Figure 1 depicts an example of the measurements of the six angles in a 3D volume of a premenopausal woman with an adnexal mass where the histological result was a borderline ovarian tumor

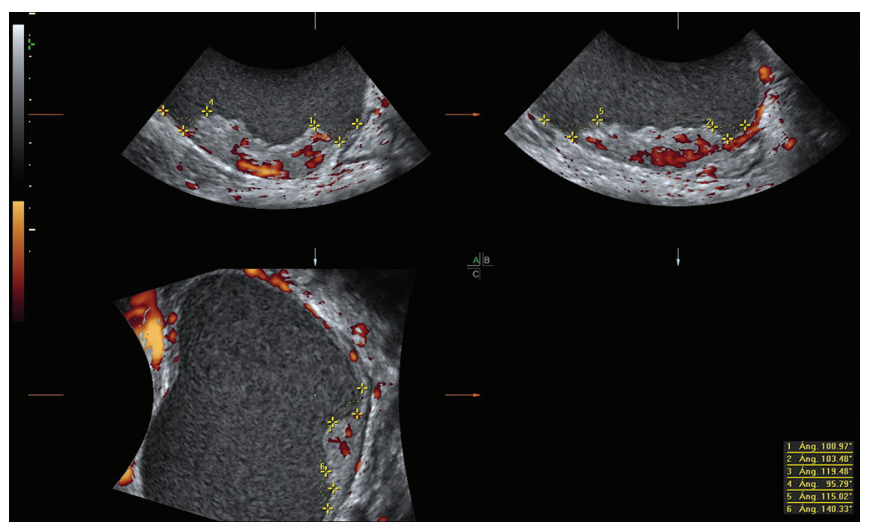

Fig. 2: All obtuse angles in a primary invasive ovarian tumor of a postmenopausal woman 
Table 1: Results of the different variables of benign and malignant masses

\begin{tabular}{|c|c|c|c|}
\hline & \multicolumn{2}{|c|}{ Definite diagnosis $(n=81)$} & \multirow[b]{2}{*}{ pvalue } \\
\hline & Benign $(n=52)$ & Malignant $(n=29)$ & \\
\hline Average angle* & $81.5^{\circ}(\mathrm{SD} 23.3,38-130)$ & $75.7^{\circ}(S D 21.0,42-116)$ & 0.270 \\
\hline Widest angle* & $105.3^{\circ}(\mathrm{SD} 25.6,59-163)$ & $103.7^{\circ}(\mathrm{SD} 28.5,62-148)$ & 0.797 \\
\hline Narrowest angle* & $58.0^{\circ}(\mathrm{SD} 23.5,22-110)$ & $49.1^{\circ}(\mathrm{SD} 19.4,23-96)$ & 0.088 \\
\hline Difference between the widest and narrowest angle* & $47.3^{\circ}(\mathrm{SD} 17.7,18-88)$ & $54.6^{\circ}(\mathrm{SD} 24.7,16-105)$ & 0.130 \\
\hline Number of obtuse angles* & $2.3(\mathrm{SD} 2.2,0-6)$ & $1.8(S D 1.9,0-6)$ & 0.249 \\
\hline Number of acute angles* & 3.7 (SD 2.2, 0-6) & 4.2 (SD 1.9,0-6) & 0.249 \\
\hline
\end{tabular}

*Standard deviation (SD) and range in parenthesis

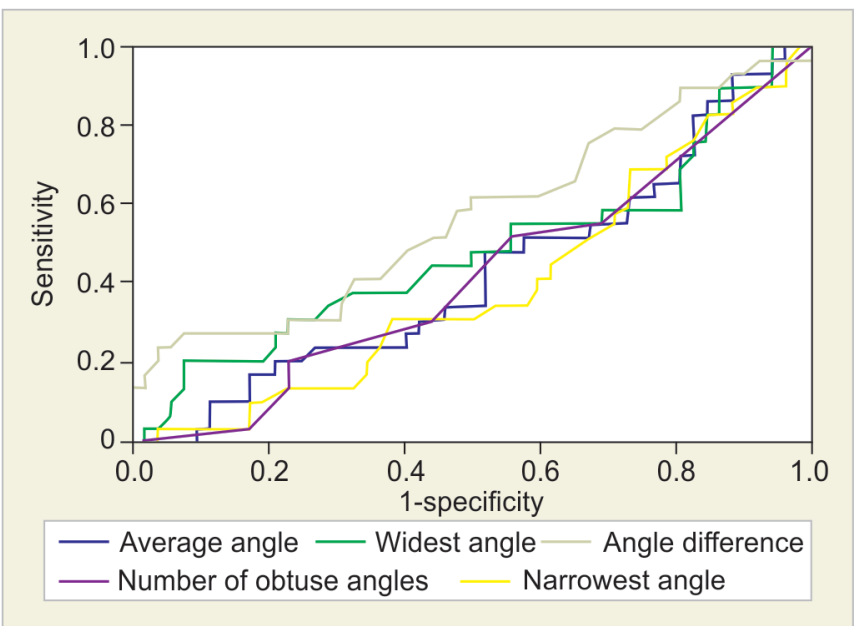

Fig. 3: ROC curves: average angle, widest angle, narrowest angle, the difference between the widest and narrowest angle, and the number of obtuse angles

which's angles were all acute, with a widest and narrowest angle of $75.9^{\circ}$ and $41.1^{\circ}$, respectively, and average angle of $61.7^{\circ}$. Figure 2 corresponds to a 3D volume of a postmenopausal patient with a primary invasive ovarian tumor confirmed by histology, all the angles of the solid component were obtuse, the widest and narrowest angle were $140.3^{\circ}$ and $95.8^{\circ}$, respectively, and the average angle of $112.5^{\circ}$.

The ROC curves are shown in Figure 3. The area under the curve (AUC) of average angle, widest angle, narrowest angle, difference between the widest and narrowest angle, and the number of obtuse angles were $0.427(95 \% \mathrm{Cl} 0.29-0.557, p=0.281), 0.481(95 \% \mathrm{Cl}$ $0.342-0.620, p=0.799), 0.395$ (95\% Cl 0.268-0.521, $p=0.118), 0.572$ (95\% Cl 0.437-0.706, $p=0.287$ ), and 0.421 (95\% Cl 0.292-0.550, $p=0.241)$, respectively.

\section{Discussion}

The results of this study show that the measurement of the angles between the solid component and the cyst's wall of a unilocularsolid adnexal mass with only one papillary projection is not useful for discriminating malignant from benign masses. Even with different approaches to this angle, such as, average angle, widest angle, narrowest angle, the difference between the widest and narrowest angle, number of obtuse and acute angles we found the measurement of the angle is not useful.
Most of the tumors classified as unilocular-solid cyst were benign, but the number of malignant was not small, of which the majority were primary invasive ovarian tumors, showing that this sonographic characteristic needs to be analyzed more carefully with power Doppler, morphological assessment, and complementary studies or surgical approach in case of doubt because of the higher risk of malignancy compared to unilocular cists without solid component. ${ }^{15,16}$

On the one hand, Hassen et al. found that the presence of an obtuse angle within a papillary projection confers a greater risk of malignancy with an OR of 11.07 (95\% Cl 2.51-48.84, $p=0.001){ }^{11}$ which differs from our results were none of the analyzed variables were statistically significant. On the other hand, Landolfo et al. results match ours by stating that the presence of obtuse angles does not seem to help in the differentiation of malignant and benign tumors $(p=0.21)^{12}$

The main difference between this study and the previous is that we measured the angle in the two lateral borders of the papillary projection, in a three-dimensional volume, with three planes, obtaining six different values, whereas Hassen et al. and Landolfo et al. measured both lateral angles of the solid component in a twodimensional image. ${ }^{11,12}$ Furthermore, they analyzed these values as a dichotomous variable describing it only as acute or obtuse, whereas the present study uses an absolute value for the angle turning it into a quantitative variable. In addition, the previously mentioned researches included masses with one or more than one papillary projections, whereas we only included masses with only one papillary projection.

Therefore, the studies are not quite comparable because of the different approach of the measurement and the differences between the studied populations. Our patients were highly selected from a series of women with unilocular-solid cysts with only one papillary projection and without peritoneal carcinomatosis or ascites, whereas in the other researches these patients were included, possibly explaining the differences in the results. The prevalence of malignant tumors in our series was identical to that reported by Landolfo et al. (35.8\%), but with a difference in the distribution of malignancies were different. In Landolfo's series, $20.6 \%$ of all tumors corresponded to borderline tumors (8.6\% in our study) and $15.2 \%$ primary invasive or metastatic tumors $(25.9 \%$ in our study). ${ }^{12}$ Hassen et al. reported a rate of malignancy of $62.7 \%$, where $16.9 \%$ corresponded to borderline tumors and $45.8 \%$ to malignant tumors. ${ }^{11}$

To our knowledge, this is the first paper that uses a different approach in the measurement of the angle between the papillary projection and cyst's wall, with a more thorough evaluation of all 
the possible angles, taking into account the different orthogonal planes that can be obtained while evaluating an adnexal mass, unlike other authors that only use a two-dimensional plane. There is limited literature regarding specific morphological characteristics in unilocular-solid cysts, specifically the angle measurement and this paper analyzes it in depth.

The limitations of this study are that it has a relatively small number of patients with a highly selected population so that these data cannot be generalized. The results can only be applied to unilocular-solid cysts with only one papillary projection, and patients without ascites or peritoneal carcinomatosis where the prevalence of malignancy would be greater than the one shown in this series.

Another limitation is that we did not assess interobserver variability. Therefore, it is not known how reproducible these measures are.

Furthermore, we did not analyze other specific parameters in these lesions, such as, the surface and size of the solid component or the presence of vascular flow with Doppler within the papillary projection which have shown to have more diagnostic value while discriminating malignant from benign masses. ${ }^{10,17,18}$ Juez et al. showed that the morphological characteristics that can be more frequently associated with malignancy were the larger tumor size, irregular surface, and abundant color score. ${ }^{18}$ The purpose of this study was specifically to analyze this parameter of the angle in the solid component without taking into account the other morphological characteristics to evaluate if it could help in the diagnosis.

\section{Conclusion}

Measurement of the angles between the solid component and the cyst's wall in unilocular-solid adnexal masses with only one papillary projection is not useful as a predictor of malignancy.

\section{References}

1. Timmerman $D$, Schwärzler $P$, Collins WP, et al. Subjective assessment of adnexal masses with the use of ultrasonography: an analysis of interobserver variability and experience. Ultrasound Obstet Gynecol 1999;13(1):11-16. DOI: 10.1046/j.1469-0705.1999.13010011.x.

2. Valentin L, Hagen B, Tingulstad S, et al. Comparison of 'pattern recognition' and logistic regression models for discrimination between benign and malignant pelvic masses: a prospective cross validation. Ultrasound Obstet Gynecol 2001;18(4):357-365. DOI: 10.1046/j.0960-7692.2001.00500.x.

3. Sokalska A, Timmerman D, Testa AC, et al. Diagnostic accuracy of transvaginal ultrasound examination for assigning a specific diagnosis to adnexal masses. Ultrasound Obstet Gynecol 2009;34(4):462-470. DOI: $10.1002 /$ uog.6444.

4. Alcázar JL, Guerriero S, Laparte C, et al. Diagnostic performance of transvaginal gray-scale ultrasound for specific diagnosis of benign ovarian cysts in relation to menopausal status. Maturitas 2011;68(2):182-188. DOI: 10.1016/j.maturitas.2010.09.013.

5. Valentin L, Ameye L, Jurkovic D, et al. Which extrauterine pelvic masses are difficult to correctly classify as benign or malignant on the basis of ultrasound findings and is there a way of making a correct diagnosis? Ultrasound Obstet Gynecol 2006;27(4):438-444. DOI: 10.1002/uog.2707.

6. Valentin L. Pattern recognition of pelvic masses by gray-scale ultrasound imaging: the contribution of Doppler ultrasound. Ultrasound Obstet Gynecol 1999;14(5):338-347. DOI: 10.1046/j.14690705.1999.14050338.x.

7. Ameye L, Timmerman D, Valentin L, et al. Clinically oriented three-step strategy for assessment of adnexal pathology. Ultrasound Obstet Gynecol 2012;40(5):582-591. DOI: 10.1002/uog.11177.

8. Timmerman D, Testa AC, Bourne T, et al. Simple ultrasound-based rules for the diagnosis of ovarian cancer. Ultrasound Obstet Gynecol 2008;31(6):681-690. DOI: 10.1002/uog.5365.

9. Valentin L, Ameye L, Savelli L, et al. Adnexal masses difficult to classify as benign or malignant using subjective assessment of gray-scale and Doppler ultrasound findings: Logistic regression models do not help. Ultrasound Obstet Gynecol 2011;38(4):456-465. DOI: 10.1002/ uog.9030.

10. Valentin L, Ameye L, Savelli L, et al. Unilocular adnexal cysts with papillary projections but no other solid components: Is there a diagnostic method that can classify them reliably as benign or malignant before surgery? Ultrasound Obstet Gynecol 2013;41(5):570-581. DOI: 10.1002/uog.12294.

11. Hassen K, Ghossain MA, Rousset P, et al. Characterization of papillary projections in benign versus borderline and malignant ovarian masses on conventional and color Doppler ultrasound. AJR Am J Roentgenol 2011;196(6):1444-1449. DOI: 10.2214/AJR. 10.5014.

12. Landolfo $C$, Valentin $L$, Franchi $D$, et al. Differences in ultrasound features of papillations in unilocular-solid adnexal cysts: a retrospective international multicenter study. Ultrasound Obstet Gynecol 2018;52(2):269-278. DOI: 10.1002/uog.18951.

13. Alcázar JL, García-Manero M, Galván R. Three-dimensional sonographic morphologic assessment of adnexal masses: a reproducibility study. J Ultrasound Med 2007;26(8):1007-1011. DOI: 10.7863/jum.2007.26.8.1007.

14. Timmerman $D$, Valentin $L$, Bourne $T H$, et al. International Ovarian Tumor Analysis (IOTA) group. Terms, definitions and measurements to describe the sonographic features of adnexal tumors: a consensus opinion from the international ovarian tumor analysis (IOTA) group. Ultrasound Obstet Gynecol 2000;16(5):500-505. DOI: 10.1046/j.14690705.2000.00287.x.

15. Ekerhovd E, Wienerroith $\mathrm{H}$, Staudach $\mathrm{A}$, et al. Preoperative assessment of unilocular adnexal cysts by transvaginal ultrasonography: a comparison between ultrasonographic morphologic imaging and histopathologic diagnosis. Am J Obstet Gynecol 2001;184(2):48-54. DOI: $10.1067 / \mathrm{mob} .2001 .108330$.

16. Sayasneh A, Ekechi C, Ferrara L, et al. The characteristic ultrasound features of specific types of ovarian pathology (review). Int J Oncol 2015;46(2):445-458. DOI: 10.3892/ijo.2014.2764.

17. Fagotti $A$, Ludovisi $M$, De Blasis I, et al. The sonographic prediction of invasive carcinoma in unilocular-solid ovarian cysts in premenopausal patients: a pilot study. Hum Reprod 2012;27(9):2676-2683. DOI: 10.1093/humrep/des231.

18. Juez L, Peces A, Martines-Astorquiza Corral T, et al. Ultrasound features for determining the risk of malignancy in unilocularsolid adnexal masses in premenopausal women without ascites and/or carcinomatosis. Donald School J Ultrasound Obstet Gynecol 2015;9(2):112-117. DOI: 10.5005/jp-journals-100091398. 